Service social

\title{
Intervention de groupe auprès d'enfants de parents divorcés
}

\section{Isabelle Côté}

Volume 37, numéro 1-2, 1988

Par-delà les barrières des sexes

URI : https://id.erudit.org/iderudit/706393ar

DOI : https://doi.org/10.7202/706393ar

Aller au sommaire du numéro

\section{Éditeur(s)}

École de service social de l'Université Laval

\section{ISSN}

1708-1734 (numérique)

Découvrir la revue

\section{Citer ce document}

Côté, I. (1988). Intervention de groupe auprès d'enfants de parents divorcés. Service social, 37(1-2), 214-223. https://doi.org/10.7202/706393ar

\section{Résumé de l'article}

Précisant au départ quelques éléments du contexte social et organisationnel, l'auteure décrit une intervention de groupe auprès d'enfants de parents divorcés. Ce projet a ceci de particulier que deux sessions (l'une pour les parents, l'autre pour les enfants) sont menées simultanément. À partir des données recueillies sur les effets du projet et sur la satisfaction des participants, des pistes de recherche et des suggestions pour l'intervention sont dégagées. 


\section{COMMENTAIRES \\ ET DOCUMENTS}

COrÉ, Isabelle, travailleuse sociale au C.L.S.C. Sainte-Foy Sillery.

\section{Intervention de groupe auprès d'enfants de parents divorcés}

\section{Isabelle Côté}

Les conséquences du divorce sur les enfants préoccupent tout autant les parents que les enseignants et les intervenants. Que dire aux enfants? Comment les aider à s'exprimer? Resteront-ils marqués par cet événement?

Si les parents ont le droit de divorcer, d'exprimer leurs difficultés, d'être compris et aidés par leurs amis et par leur milieu, les enfants ontils les mêmes possibilités et moyens?

Avec un taux de divorce qui continue d'augmenter, ces questions sont très pertinentes. Au Québec, on comptait en 1969 environ un divorce pour onze mariages, alors qu'en 1982 cette proportion était passée à un divorce pour deux mariages. ${ }^{1}$ Quels que soient les chiffres, la séparation des parents représente pour tout enfant une expérience subjective qui n'a rien à voir avec les statistiques.

C'est à partir de ce constat, tiré de notre pratique professionnelle et de la littérature, que nous avons mis sur pied au Centre local de services communautaires Sainte-Foy Sillery un projet d'intervention de groupe, à l'intention des parents et de leurs enfants confrontés à cette nouvelle situation de vie. Le présent article porte sur cette intervention en insistant sur l'expérimentation auprès des jeunes. 


\section{Historique de l'intervention}

Implanté à partir d'avril 1985, ce C.L.S.C. est préoccupé d'offrir, en milieu ouvert, des services qui rejoignent vraiment les besoins de la population. Sensibilisé au fait que le divorce vient au deuxième rang, immédiatement après le décès d'un conjoint, parmi les événements générateurs de stress ${ }^{2}$ chez les adultes et chez les enfants, cet organisme a mis sur pied, en septembre 1985, un groupe d'intervention auprès des personnes confrontées à cette réalité.

Cette décision s'est avérée très opportune puisque, un an plus tard, une étude effectuée auprès de la clientèle du secteur Famille-Jeunesse ${ }^{3}$ révélait que $38 \%$ de la clientèle est séparée (légalement ou de fait) ou divorcée. Ces clients demandent d'être aidés surtout à cause de la séparation du couple et expriment leur besoin d'être supportés dans leurs relations avec leurs enfants.

\section{Objectifs}

Les objectifs du groupe d'intervention sont les mêmes pour les parents et les enfants :

- briser l'isolement ;

- permettre aux parents et aux jeunes d'exprimer leurs sentiments et leur vécu par rapport au divorce ;

- les aider à normaliser leurs sentiments et réactions, avec l'appui d'un contenu théorique ;

- créer un réseau d'entraide entre pairs, susceptible de subsister après les rencontres ;

- faciliter le dialogue parents-enfants au sujet du divorce afin de le vivre d'une façon plus harmonieuse.

Toutefois, des objectifs spécifiques guident l'intervention auprès des jeunes:

- les aider à se dégager de la responsabilité qu'ils s'attribuent souvent face au divorce ;

- les amener à échanger sur les changements positifs et négatifs que cette situation leur fait vivre ;

- leur faire identifier leurs besoins et attentes par rapport à certaines situations liées au divorce : (v.g. le(la) nouvel(le) ami(e) du père, de la mère, le maintien du lien avec les deux parents, la position "tampon» entre ex-conjoints qui ne communiquent pas);

- les inviter à exprimer leurs préoccupations à leurs deux parents. 


\section{Hypothèses de travail}

Le projet repose sur les deux hypothèses suivantes:

- bien que les deux sessions de groupes soient distinctes (une pour les parents, une pour les enfants) elles se ressemblent quant au contenu. Nous postulons que cette similitude, vécue dans une même période de temps, active les échanges parentsenfants et favorise une adaptation plus rapide à la nouvelle situation de vie ;

- les parents nous mentionnent régulièrement, et notre pratique le confirme, que les enfants parlent peu du divorce ou s'en ouvrent avec difficulté. À ce propos, nous postulons que, regroupés par catégories d'âge (6 à 8 ans; 9 à 12 ans) dans une atmosphère de détente avec une animation adaptée à leur âge, les jeunes deviennent capables d'exprimer leurs sentiments par rapport au divorce de leurs parents et à ses conséquences pour eux.

Cette intervention s'est inspirée de deux expériences:

- la session Mieux vivre un divorce ${ }^{4}$ qui regroupait des personnes séparées ou divorcées depuis moins d'un an ;

- une expérience américaine avec un groupe d'enfants de 7 à 12 ans dont les parents étaient séparés. ${ }^{5}$

\section{Modèle d'intervention}

En conformité avec le projet mixte d'intervention, nous avons privilégié une combinaison de deux modèles: la réciprocité et l'éducation.

Le modèle de réciprocité ${ }^{6}$ présente les différentes parties des systèmes concernés comme étant en interaction (parents-enfants-organisme-intervenant) et définit le rôle du travailleur social comme pouvant améliorer l'interaction entre les divers systèmes; il permet aussi de travailler les transactions du groupe, ici et maintenant. Le travail de groupe y est privilégié pour aider à la résolution d'un problème. Un concept clé de ce modèle est l'aide mutuelle entre les membres, incluant aussi l'intervenant social. Le groupe est une condition nécessaire à la résolution d'un problème.

Le modèle d'éducation permet, de son côté, au praticien social de partager certaines données théoriques sur le divorce afin d'aider parents et enfants à mieux intégrer leur vécu, à le normaliser. 
Ces deux modèles donnent la possibilité d'intervenir en situation de crise. Une intervention minimale suffit parfois pour redonner à une personne les moyens de faire une première intégration et faciliter son adaptation à la nouvelle situation. ${ }^{7}$

\section{Évolution du modèle d'intervention auprès des adultes}

Dans un contexte où le divorce était peu courant, la session Mieux vivre un divorce fut d'abord dispensée sous une forme didactique. À ce moment, les personnes récemment divorcées avaient besoin d'être rassurées par des éléments théoriques, tant au plan personnel que parental.

En régions urbaines, l'augmentation fulgurante du nombre de divorces a eu pour effet d'accélérer le processus d'information sur le sujet. Actuellement, les besoins sont donc moins de recevoir un enseignement que de partager un vécu afin d'assumer cette tâche psychologique liée au divorce. Ayant animé six sessions Mieux vivre son divorce (1980-1982), la responsable de notre projet souhaitait prolonger l'expérience tout en développant le caractère existentiel du programme. De plus, elle voulait tenir compte de la demande de plusieurs participants qui soulignaient la nécessité de rencontres semblables pour leurs enfants.

Depuis 1985, donc, la session est offerte à un nombre restreint de parents (sept à neuf) ayant ou non la garde de leurs enfants.

Quatre postulats de base guident les huit rencontres :

- tout en étant une difficile étape de transition, le divorce, comme toute crise, peut être source de croissance ;

- le bien-être de l'enfant passe par le bien-être de ses parents, surtout de celui qui en a la garde. De là l'importance pour l'adulte de se donner les moyens d'assumer cette situation;

- le divorce signifie la fin du couple mais non celle du système familial. En ce sens, une importante tâche de réorganisation s'impose ;

- le maintien du lien avec ses parents peut aider l'enfant à vivre plus positivement le divorce.

Pour atteindre l'objectif interactionnel des deux projets de groupe, nous comptons d'abord et avant tout sur les parents. En effet, dès qu'ils s'inscrivent à la session adulte, les parents sont informés de la possibilité d'une session pour leurs enfants. S'ils sont intéressés à cette forme d'aide pour leurs enfants, nous leur demandons d'informer ceux-ci d'une session éventuelle juste pour eux, tout en respectant leur droit 
d'y participer ou non. C'est à eux aussi que revient la responsabilité de sensibiliser l'autre parent à cette possibilité d'aide offerte à leur enfant.

Lors de la première rencontre avec les adultes, les animatrices leur expliquent que leurs enfants seront très sensibles à évaluer, à leur façon, les réactions de leurs parents à la session à laquelle ceux-ci participent. On leur dit aussi qu'ils sont des motivateurs capables de prédisposer positivement leur enfant à l'invitation téléphonique qui leur sera faite par une animatrice deux ou trois semaines plus tard. Effectivement, lors des appels, les jeunes nous tiennent les propos suivants :

"Maman est moins triste depuis qu'elle va à son groupe, je suis gênée mais peut-être que ça va être le fun pour moi aussi. " (Josiane, 9 ans)

"Maman m'a dit qu'elle s'était fait des amis et que moi aussi je pourrais m'en faire. " (Vincent, 8 ans)

"Il paraît qu'on dit nos sentiments et que ça fait du bien. " (Julie, 11 ans)

À ce moment-ci, parents et jeunes savent déjà que, dans leur groupe respectif, ils aborderont les mêmes thèmes (sentiments reliés à la séparation, estime de soi, vie relationnelle, contacts parents-enfants, etc.), dans un cadre distinct et avec des moyens différents toutefois.

\section{Intervention de groupe auprès des enfants : cadre physique et aspects techniques}

Pour les jeunes, nous avons privilégié, de façon encore plus sentie, une atmosphère propice à la détente, à la complicité, aux confidences, afin qu'ils se sentent à l'aise de parler entre eux et avec les animatrices de la séparation ou du divorce de leurs parents. Les rencontres ont lieu dans un petit local du C.L.S.C. agréablement décoré où, assis sur des coussins, jeunes et animatrices peuvent échanger de façon plus décontractée sur un sujet pourtant plein d'émotion.

Chaque groupe réunit quatre à six participants(es) et deux animatrices. Ce petit nombre de personnes facilite l'échange et en renforce le caractère intimiste. La session compte un maximum de quatre rencontres dont la durée varie d'après l'âge : 90 minutes pour les 6-8 ans, deux heures pour les 9-12 ans, deux heures et demie pour les adolescents.

Nos instruments d'animation sont nombreux:

- jeux variés pour faire connaissance, reprendre contact au début de chaque rencontre;

- livre qui traite du divorce des parents; les enfants de 5 à 12 ans peuvent le lire, le colorier, le faire lire à l'un ou l'autre de leurs parents ; 
- vidéos : quelques-uns extraits de la série À plein temps, comme Sylvie agent-double ou La nouvelle blonde de mon père. Les adolescents sont fort intéressés par le vidéo Les enfants divorcentils? ;

- dessins (de leur famille, de leur réseau, de leurs rêves);

- photos de famille;

- marionnettes, pour rejoindre les 6 à 8 ans.

Les marionnettes ont servi à créer deux personnages: Sally, une fillette de 7 ans et son petit frère Martin, 5 ans. Compte tenu qu'on nous demande souvent comment nous utilisons les marionnettes, nous nous attarderons davantage à cet outil d'animation. Les animatrices construisent des scénarios appropriés aux enfants de cet âge qui, par leurs réparties, alimentent l'échange, activité qui peut facilement durer de 15 à 20 minutes et même se continuer à un autre moment de la rencontre. Une animatrice manie les marionnettes, tandis que l'autre anime les enfants dans leurs répliques à Sally et à Martin. Elle décrit à la marionnette le non-verbal des enfants afin que cette forme de communication soit utilisée au maximum. Elle fait aussi une synthèse théorique de l'interaction en cours. II s'agit-là de moments privilégiés où le ici et maintenant prend tout son sens.

Voici un exemple de scénario et d'échange marionnettes-enfants.

\section{Thème : L'importance du père (scénario de la deuxième rencontre)}

Sally remet aux enfants le dessin qu'elle a fait la semaine précédente. On y voit Sally et Martin dans une maison (celle de leur mère) et qui se préparent en vue de rejoindre leur père. Sally dit qu'elle est triste et un des enfants, Francis, dit qu'il l'est aussi.

L'animatrice demande aux enfants ce que signifie le dessin. Sylvain ( 8 ans) répond : "Ça veut dire qu'on peut toujours aller voir son père ". Les autres enfants abondent dans le même sens, sauf Julie (7 ans), qui dit: "Mon père demeure loin-loin, je ne le vois pas souvent". Sally intervient alors et dit à Julie avec tristesse : "Mon père ne demeure pas loin-loin mais je ne le vois pas souvent". Martin est fâché : "Il avait promis de venir dimanche et il n'est pas venu ".

Les enfants réconfortent les deux marionnettes, l'animatrice fait de même et demande aux enfants : "Est-ce que cela vous est déjà arrivé ? "

"Oui, c'est parce qu'il travaillait." (Johathan, 7 ans).

"Non, mais ma mère n'a pas voulu que j'y aille une fois, j'y suis allé quand même." (Sylvain, 8 ans) 
"Non et je suis contente parce que je le verrais encore moins. " (Julie, 7 ans)

L'animatrice demande à Sally et Martin s'ils savent pourquoi leur père n'est pas venu. Ils répondent par la négative. Elle demande aux enfants de suggérer des raisons à Sally et Martin :

"Peut-être qu'il travaillait."

"Il a peut-être oublié."

"Il n'y avait pas de téléphone où il se trouvait. "

Les enfants sentent le besoin de rassurer Sally et Martin : "Tu vas revoir ton papa, il t'aime ". Sally et Martin disent que ça leur fait du bien d'en parler avec eux et promettent de revenir les voir.

L'animatrice revient sur cette situation et facilite l'expression des sentiments autour de cette question; elle renforce le support apporté par les enfants et ajoute qu'ils ont le droit de continuer à aimer leurs parents même après la séparation. Elle cherche avec eux des moyens pour maintenir un lien avec un parent (souvent le père) qu'on ne voit pas souvent. Les suggestions fusent: "Lui envoyer des dessins, des lettres, lui téléphoner (demander la permission à maman si c'est un interurbain)."

Les rencontres se déroulent rapidement et intensément. Elles sont ponctuées d'une pause-gâterie (collation) et les enfants de 5 à 12 ans reçoivent toujours une petite surprise à la fin de la soirée (gomme à effacer de collection, autocollant, etc.). Quant aux tout-petits, ce sont les marionnettes qui leur remettent le cadeau. II nous apparaît important de mentionner que la même règle de confidentialité vaut pour tous les groupes. Les enfants sont libres de raconter, à leur manière, ce qu'ils ont retenu de leur soirée. Ainsi, les parents sont invités à ne pas trop questionner leurs enfants, à les écouter s'ils relatent certains faits ou propos, et surtout à répondre aux questions de clarification, s'il y a lieu. Jusqu'à présent la confidentialité a été respectée, tant par les parents que par les enfants, à la satisfaction de tous.

\section{Évaluation}

Depuis septembre 1985, sept groupes de parents (40 adultes: 33 femmes, 7 hommes) et sept d'enfants ( 39 jeunes : 23 garçons, 16 filles) ont pu participer au projet. II y a eu deux groupes d'enfants de 5 à 8 ans, 4 de 9 à 12 ans et un avec 5 adolescents de 15 à 17 ans. En tout, deux adultes ont abandonné et un seul enfant. Le taux de participation aux rencontres est de $90 \%$ chez les adultes et de presque $100 \%$ chez les enfants. 
En fin de session, les adultes ont eu à répondre à un questionnaire écrit. Outre leur appréciation fort favorable pour l'ensemble des rencontres, ils ont unanimement indiqué qu'ils avaient beaucoup apprécié qu'une session soit offerte à leurs enfants ou adolescents et souhaitaient que cette initiative se continue.

Nous avons aussi effectué une rencontre de relance avec trois groupes adultes et le groupe d'adolescents, six mois après la fin des sessions respectives. Le taux de participation a été de $85 \%$ pour les adultes et de $\mathbf{1 0 0} \%$ pour les adolescents. Voici quelques commentaires des adultes :

"Cette session n'a pas tout réglé mais elle m'a permis de faire un bout aux plans personnel et parental. ”

" Je me sens moins seul(e) dans cette situation, je n'ai plus l'impression d'avoir le mot séparation inscrit sur le front."

"Mon enfant (10 ans) jasait plus du divorce avec moi pendant qu'il allait à ses rencontres. À un moment, il m'a dit: "Maintenant c'est assez, je veux qu'on parle d'autre chose." Je crois qu'il a bien raison. J'ai continué à avoir des contacts avec deux personnes du groupe, nos enfants aussi se voient. "

" Certains disaient aussi que l'ex-conjoint(e) s'impliquait plus dans la tâche parentale, qu'il y avait moins d'agressivité dans l'air."

Ces extraits montrent bien les gains que la majorité des participants ont pu obtenir à suivre nos programmes. Tous n'en retirent pas les mêmes avantages; quelques-uns doivent poursuivre une démarche de consultation individuelle, et d'autres restent " accrochés " à une phase ou l'autre de la séparation (négation, agressivité, culpabilité, etc.) mais, dans l'ensemble le bilan est positif.

Quant aux adolescents, ils mentionnaient :

être passé à autre chose ;

qu'ils se sentaient moins mal à l'aise quand leur parent (la mère) pleurait ;

qu'ils avaient aimé que les rencontres se tiennent au C.L.S.C. plutôt qu'à l'école parce qu'ils étaient plus certains de la confidentialité. nément :

Quant aux enfants, ils donnent leur appréciation très sponta-

"C'était l'fun, le local était beau et j'ai aimé les surprises. "

"Pourquoi nous, on a juste quatre rencontres et les grands en ont huit, c'est pas juste. " (plusieurs enfants)

« J'aurais jamais pensé que presque tout le monde déménageait après une séparation. $\nsim$ (Cathy, 11 ans) 
"Ça fait du bien de dire ce qu'on ressent, j'ai parlé davantage à mon père et à ma mère. Ce n'est pas à cause de moi qu'ils se sont séparés. » (Emmanuel, 10 ans)

"Les animatrices sont fines, pis ça ressemble pas trop à l'école." (Sylvain, 8 ans)

"Est-ce qu'on va se revoir? " (la grande majorité des enfants)

Bien que fragmentaire et non rigoureuse, cette évaluation nous permet de constater que le projet rejoint les objectifs formulés au début de cet article, tant pour les adultes que pour les enfants. De plus, des parents non inscrits mais qui avaient accepté que leurs enfants participent ont communiqué avec nous pour nous donner leur appréciation et expliquer aussi qu'au début, ils avaient peur, craignaient que le fait de parler de la séparation ne bouleverse leurs enfants davantage. D'autres nous ont confié qu'ils faisaient plus d'efforts pour parler à leur exconjoint(e) pour s'entendre de façon plus harmonieuse sur les sorties et les visites. D'ailleurs, certains se sont inscrits pour une session ultérieure.

À la lumière de l'évaluation, nous constatons que la complémentarité d'intervention auprès des parents et des enfants, tant dans le temps que dans les thèmes abordés, s'est avérée efficace. Réfléchir dans des groupes séparés, mais complémentaires par leurs tâches psychologiques, intensifie le dialogue entre les deux parties et crée une synergie positive propre à amplifier les effets bénéfiques du dialogue. En somme, chacun et chacune apprennent à parler et à se parler du divorce dans un créneau positif.

\section{Suggestions et perspectives de recherche}

Comme phénomène social, le divorce est là pour rester et, puisque le projet ici décrit a reçu un accueil fort positif des parents et de leurs enfants, le C.L.S.C. Sainte-Foy Sillery entend poursuivre l'expérimentation. Les intervenantes veulent apporter des modifications susceptibles d'élargir quelque peu l'accès à la session de groupe pour enfants. On intégrera quelques enfants dont les parents ont un suivi individuel et qui sont divorcés depuis plus ou moins longtemps. Nous croyons que l'homogénéité du groupe quant au temps de séparation ou de divorce n'a pas, pour les enfants, la même importance que pour les adultes.

Nous souhaitons qu'une telle expérience se vive dans d'autres milieux, incluant entre autres les C.L.S.C. Déjà, un organisme familial privé compte l'offrir à une clientèle semi-urbaine; le projet a aussi essaimé en milieu rural avec quelques variations. Nous croyons essentiel de souligner que ce type de projet comporte des exigences précises: une très bonne connaissance théorique et pratique de la problématique 
du divorce, tant pour les adultes que pour les enfants, une expérience professionnelle en groupe et une capacité à composer avec l'imaginaire des enfants.

Il serait intéressant qu'une recherche éventuelle évalue l'impact de ce type d'intervention interactionnelle sur le maintien du lien de l'enfant avec ses parents, suite à une rupture du couple.

\section{Références}

1 Bureau de la statistique du Québec (B.S.Q.), Démographie québécoise : passé, présent, perspectives, Québec, B.S.Q., 1983.

2 Holmes, T.H. et R.H. RAHE, "The social readjustment rating scale", Journal of Psychosomatic Research, vol. 11, 1967, p. 213.

3 TARD, Caroline, Analyse de la clientèle de l'équipe Famille-Jeunesse du Centre local de services communautaires Ste-Foy-Sillery en implantation, Québec, Centre de recherche sur les services communautaires, Université Laval, octobre 1986, 29p.

4 Poirier, Gilles et Louise TURgeon, Pour mieux vivre un divorce : session avec des personnes récemment séparées ", Intervention, n० 61, 1981 : 17-22.

5 Bonkowski, Sara E., Shelly Q. Beguette et Sara Boomhower, "A group design to help children adjust to parental divorce", Social Casework, vol.65, no 3, 1984 : 131-136.

6 Schulman, L., The Skills of Helping Individuals and Groups, Itasca (III.), Peacock Publishers, 1979.

7 Poirier, Gilles et Louise Turgeon, op. cit.

\section{Bibliographie complémentaire}

BIRD-FranCKE, Linda, Les enfants face au divorce, Paris, Robert Laffont, 1986, 243p. ("Réponses".)

GARDNER, Richard A., Le divorce expliqué aux filles et aux garçons, Montréal, Sélect, 1978, 157p.

POIRIER, Gilles, Les enfants et le divorce, une revue de littérature, Service de la recherche et de la documentation, Centre de services sociaux de Québec, 1981, 142p.

RoBson, Bonnie, Les enfants du divorce se racontent, Montréal, Éditions du Jour, $1980,175 p$.

Wallenstein, Judith et Joan Kelly, Surviving the Break-Up: How Children and Parents Cope with Divorce, New York, Basic Books, 1980, 341p. 